\title{
Antibacterial Efficacy of Neem, Triphala, Green Tea, and Combination of Neem with Triphala Extract against Enterococcus faecalis: An in vitro Study
}

\author{
${ }^{1}$ Abhinav C Singhal, ${ }^{2}$ Aditi S Jain, ${ }^{3}$ Jayesh Tiwari, ${ }^{4}$ Pratik Surana, ${ }^{5}$ Neha Saini
}

\begin{abstract}
Introduction: The main objective of root canal treatment is to disinfect the entire root canal system, and irrigation is an important step in reducing the bacterial load from the root canal system. However, irrigants currently used in the field of endodontics have their share of limitations, and the search for an ideal root canal irrigant continues. The use of herbal extracts as endodontic irrigants is today gaining popularity.
\end{abstract}

Aims and objectives: To evaluate and compare the antimicrobial potential of herbal extracts, such as neem (Azadirachta indica), triphala (Terminalia chebula), green tea (Camellia sinensis), and combination of neem and triphala as endodontic irrigants against Enterococcus faecalis.

Materials and methods: Freshly prepared extracts of neem, triphala, green tea, and $2 \%$ chlorhexidine were used to assess the antimicrobial efficiency against $E$. faecalis using the agar well diffusion test. Agar plates were incubated at $370^{\circ} \mathrm{C}$ for 24 hours in an incubator. The diameter of bacterial inhibition zones around each well was recorded to the nearest size in $\mathrm{mm}$.

Results: Higher mean zone of inhibition was recorded in chlorhexidine followed by neem extract and combination of neem and triphala than triphala and green tea extract respectively. The lowest mean zone of inhibition was found in green tea extract.

Conclusion: From the present study, it can be concluded that neem leaf extract shows comparable zones of inhibition with that of chlorhexidine and combination of neem and triphala.

Keywords: Antimicrobial, Enterococcus faecalis, Herbal irrigants.

How to cite this article: Singhal AC, Jain AS, Tiwari J, Surana P, Saini N. Antibacterial Efficacy of Neem, Triphala, Green Tea, and Combination of Neem with Triphala Extract against Enterococcus faecalis: An in vitro Study. Int J Experiment Dent Sci 2017;6(2):65-67.

\footnotetext{
${ }^{1,2}$ Senior Lecturer, ${ }^{3-5}$ Postgraduate Student

1,3,4 Department of Pedodontics and Preventive Dentistry, Maitri College of Dentistry \& Research Centre, Durg, Chhattisgarh India

${ }^{2}$ Department of Conservative Dentistry and Endodontics, Maitri College of Dentistry \& Research Center, Durg, Chhattisgarh India

${ }^{5}$ Department of Conservative Dentistry and Endodontics, Rungta Dental College, Bhilai, Chhattisgarh, India

Corresponding Author: Aditi S Jain, Senior Lecturer, Department of Conservative Dentistry and Endodontics, Maitri College of Dentistry \& Research Center, Durg, Chhattisgarh, India, Phone: +919425516882, e-mail: aditijain300789@gmail.com
}

\section{Source of support: Nil}

Conflict of interest: None

\section{INTRODUCTION}

The successful outcome of endodontic therapy strongly demands the complete elimination of microorganisms from the root canal system. Primary root canal infection is due to microorganisms colonizing in the pulp tissue. ${ }^{1}$ There is an abundant number of bacterial species that have been observed as inhabitants of the oral microflora, because of high nutrient availability, low oxygen potentials, and bacterial interactions in root canals, but the number of bacterial species present in endodontic infections is limited. ${ }^{2}$ Primary endodontic infections are polymicrobial in nature and dominated by Gramnegative anaerobic rods. ${ }^{3}$ Enterococcus faecalis is one of the most resistant microorganisms that dominate the microbial ecosystem of endodontic failure cases. ${ }^{4}$

Neem or A. indica has shown to be effective against various microorganisms found in the oral microflora, such as E. faecalis and Candida albicans. ${ }^{5}$ Its excellent antibacterial properties make it a material of choice for root canal irrigation and also an alternative to harmful chemical irrigants, such as $\mathrm{NaOCl}$.

Green tea polyphenols (GTPs) are obtained from C. sinensis. ${ }^{6}$ Catechins and the flavins are present in GTPs, and they are considered as microbiologically active ingredients. ${ }^{7}$

Triphala consists of Terminalia chebula, Terminalia bellerica, and Emblica officinalis, the three medicinal plants that are dried and powdered to get an Indian Ayurvedic herbal formulation. ${ }^{8}$ It has a potential of antibacterial activity against enteric pathogens, ${ }^{9}$ and also has antiinflammatory activity.

The aim of the study was to evaluate and compare extracts of neem, triphala, and green tea with chlorhexidine as a root canal irrigant.

\section{MATERIALS AND METHODS}

\section{Preparation of Green Tea and Triphala Extract}

Freshly prepared extracts of green tea (K Patel Pvt Ltd, India) and triphala (Himalaya, India) were mixed with $2 \mathrm{~mL}$ of $10 \%$ dimethyl sulfoxide (DMSO) (MERCK 


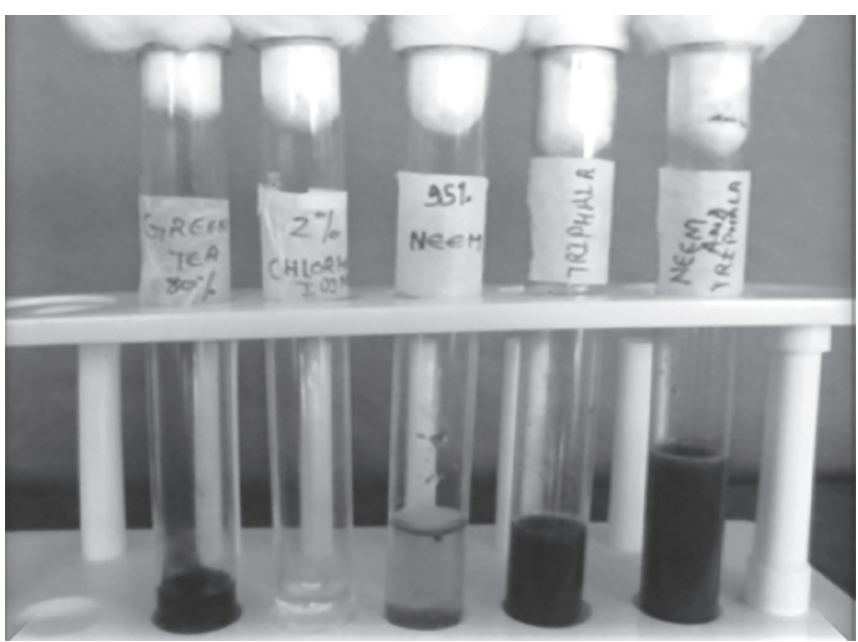

Fig. 1: Preparation of neem tree, triphala, and green tea extract

Specialty Pvt Ltd) liquid. To improve the efficiency of herbal products, DMSO is added. About $10 \%$ DMSO did not interfere with antibacterial properties was confirmed with the disk diffusion method. This was stirred for 2 minutes and then passed through fast filter paper. The strained liquid was collected and used samples were prepared (Fig. 1).

\section{Preparation of Neem Leaf Extract}

Fresh neem leaves were utilized for the study. Neem leaves were thoroughly cleaned in distilled water and afterward weighed and recorded. Around $25 \mathrm{gm}$ of fresh neem leaves was blended with $50 \mathrm{~mL}$ of absolute alcohol and the blend was macerated for 1 to 2 minutes and the partition of the course residue was done utilizing muslin cloth filtration. Same separation procedure was rehashed for the coarse residue utilizing alcohol. Both these extracted parts were later pooled together and afterward again separated utilizing fast filter paper. Up to volume of $25 \mathrm{~mL}$ was taken after separation of the alcohol from the extract and afterward the rest of the extract was stored in amber-colored airtight bottle.

\section{Agar-diffusion Test}

The bacterial stock culture E. faecalis was obtained and culture was grown overnight in brain heart infusion broth and inoculated in Mueller-Hinton agar plates. Inoculation was performed by utilizing sterile swab brushed over the media. One round well, $4 \mathrm{~mm}$ deep and $8 \mathrm{~mm}$ diameter was punched in every agar plate utilizing sterile plug-borer and the prepared irrigants were added to the wells. Agar plates were incubated at $370^{\circ} \mathrm{C}$ for 24 hours in an incubator. The diameter of bacterial inhibition zones around each well was recorded to the closest size in millimeter.

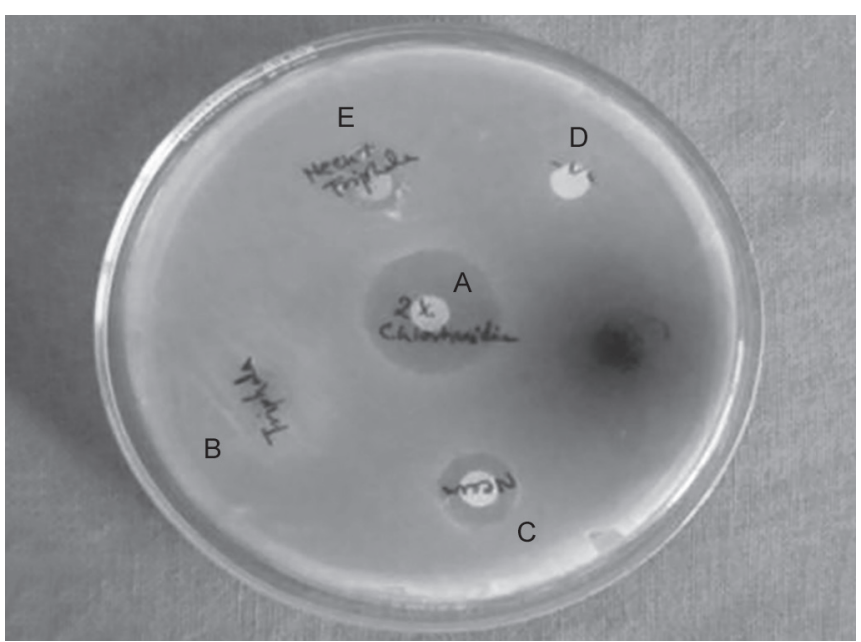

Figs 2A to E: Zones of inhibition: (A) $2 \%$ chlorhexidine; (B) triphala; (C) neem leaf extract; (D) green tea; and (E) combination of triphala and neem leaf

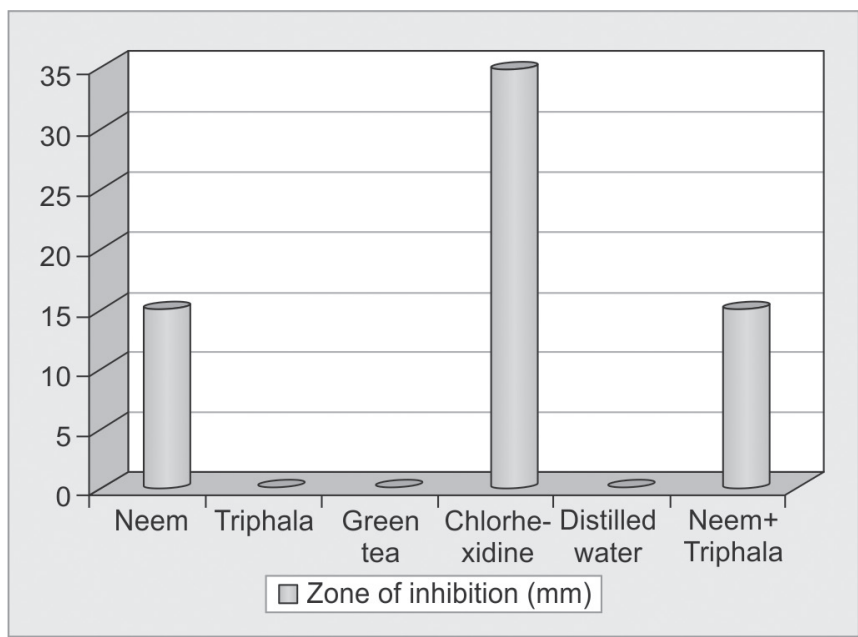

Graph 1: Comparison of the zones of inhibition of the root canal irrigants against $E$. faecalis

\section{RESULTS}

All the three medicaments showed well-defined zones of inhibition around their respective wells except the control well (Fig. 2). Analysis of variance showed significant difference between zone diameters of chlorhexidine, and neem leaf extract against $E$. faecalis ( $\mathrm{p}<0.05)$. Maximum antimicrobial activity was shown by $2 \%$ chlorhexidine, followed by neem leaf extract. Neem leaf extract showed comparable zones of inhibition to 2\% chlorhexidine (Graph 1).

\section{DISCUSSION}

Eradication of bacteria from the root canal system is important in ensuring the long-term success of root canal therapy. ${ }^{10}$ The microorganisms are the primary etiological agent in endodontic infections, and inability to destroy them influences the result of endodontic therapy. Studies have demonstrated that the bacterial flora in endodontic 
diseases is polymicrobial, with a predominance of anaerobic species. ${ }^{11,12}$ This is fundamentally accomplished through the reduction of microorganisms by debridement of the root canal system using mechanical preparation of the canals combined with chemical interventions. A few zones of the root canal walls, particularly in the apical third, are hard to clean mechanically.

Enterococcus faecalis is part of the human normal flora and an essential pathogen in opportunistic infections in humans. Enterococcus faecalis is rarely present in primary apical periodontitis, but it is the prevailing microorganism in root canal treated teeth presenting with posttreatment apical periodontitis. ${ }^{8}$ Eradication of E. faecalis from the root canal remains a challenge, as it is impervious to a variety of antimicrobial agents. Studies have also shown that E. faecalis may be one of the reasons of failure of endodontic treatments. ${ }^{13}$ The control and suppression of E. faecalis in these dental procedures are of primarily importance in decreasing the penetration of bacteria inside the dentinal tubules and furthermore restricting the development of any relationship with different microorganisms, as in virulence factors, environment, and the biofilms.

Use of neem as an endodontic irrigant might be advantageous because it is a biocompatible antioxidant, and thus not likely to cause the severe injuries to patients that might occur via $\mathrm{NaOCl}$ accidents. Bitter taste associated with this plant can be altered by different formulations due to addition of sweeteners and flavors to increase the patient compliance and acceptability. ${ }^{14}$

The results obtained in this in vitro study showed that neem leaf extract is a viable medicament against $E$. faecalis. Although $2 \%$ chlorhexidine showed comparatively more antimicrobial effect than neem, it still had an observable effectiveness against test organisms.

\section{CONCLUSION}

Neem leaf extract has a significant antimicrobial effect against E. faecalis. Preclinical and clinical trials are needed to evaluate biocompatibility before neem can conclusively be recommended as an irrigating solution, but in vitro observation of neem effectiveness appears promising.

\section{REFERENCES}

1. Vijaykumar S, GunaShekhar M, Himagiri S. In vitro effectiveness of different endodontic irrigants on the reduction of Enterococcus faecalis in root canals. J Clin Exp Dent 2010 Jul;2:e169-e172.

2. Dubey S, Chaodary M, Gupta P. Comparative study of the antimicrobial efficiency of neem leaf extract, sodium hypochlorite and biopure MTAD - an in vitro study. Indian J Dent Adv 2012 Apr;4(1):740-743.

3. Bonev B, Hooper J, Parisot J. Principles of assessing bacterial susceptibility to antibiotics using the agar diffusion method. J Antimicrob Chemother 2008 Jun;61(6):1295-1301.

4. Bhardwaj A, Velmurugan N, Sumitha, Ballal S. Efficacy of passive ultrasonic irrigation with natural irrigants (Morinda citrifolia juice, Aloe Vera and Propolis) in comparison with $1 \%$ sodium hypochlorite for removal of E. faecalis biofilm: an in vitro study. Indian J Dent Res 2013 Jan-Feb;24(1):35-41.

5. Henriques LC, de Brito LC, Tavares WL, Teles RP, Vieira LQ, Teles FR, Sobrinho AP. Microbial ecosystem analysis in root canal infections refractory to endodontic treatment. J Endod 2016 Aug;42(8):1239-1245.

6. Aw V. Discuss the role of microorganisms in the aetiology and pathogenesis of periapical disease. Aust Endod J 2016 Aug;42(2):53-59.

7. Kim SY, Shin Y, Lee CY, Jung IY. In vivo quantitative evaluation of live and dead bacteria in root canal infection by using propidium monoazide with real-time PCR. J Endod 2013 Nov;39(11):1359-1363.

8. Desai P, Himel V. Comparative safety of various intracanal irrigation systems. J Endod 2009 Apr;35(4):545-549.

9. Stuart $\mathrm{CH}$, Schwartz SA, Beeson TJ, Owatz CB. Enterococcus faecalis: its role in root canal treatment failure and current concepts in retreatment. J Endod 2006 Feb;32(2):93-98.

10. ZolettiGO, Pereira EM,Schuenck RP, Teixeira LM, Siqueira JFJr, dos Santos KR. Characterization of virulence factors and clonal diversity of Enterococcus faecalis isolates from treated dental root canals. Res Microbiol 2011 Feb-Mar;162(2):151-158.

11. Dutta A, Kundabala M. Comparative anti-microbial efficacy of Azadirachta indica irrigant with standard endodontic irrigants: a preliminary study. J Conserv Dent 2014 Mar;17(2): 133-137.

12. Tyagi SP, Sinha DJ, Garg P, Singh UP, Mishra CC, Nagpal R. Comparison of antimicrobial efficacy of propolis, Morinda citrifolia, Azadirachta indica (Neem) and 5\% sodium hypochlorite on Candida albicans biofilm formed on tooth substrate: an in vitro study. J Conserv Dent 2013 Nov;16(6):532-535.

13. Ferrer-Luque CM, Bejarano I, Ruiz-Linares M, Baca P. Reduction in Enterococcus faecalis counts-a comparison between rotary and reciprocating systems. Int Endod J 2014 Apr;47(4):380-386.

14. Stuart CH, Schwartz SA, Beeson TJ, Owatz CB. Enterococcus faecalis: its role in root canal treatment failure and current concepts in retreatment. J Endod 2006 Feb;32(2):93-98. 